\title{
Problèmes de structure dans les Andes. De la parenté, de la polygynie et des moitiés à Cuzco
}

R. Tom Zuidema

Traducteur : Marie-Claire Beauregardt et Nathan Wachtel

\section{OpenEdition \\ Journals}

Édition électronique

URL : https://journals.openedition.org/jsa/2953

DOI : 10.4000/jsa.2953

ISSN : $1957-7842$

Éditeur

Société des américanistes

Édition imprimée

Date de publication : 5 décembre 2005

Pagination : $31-49$

ISSN : 0037-9174

Référence électronique

R. Tom Zuidema, «Problèmes de structure dans les Andes. De la parenté, de la polygynie et des moitiés à Cuzco », Journal de la Société des américanistes [En ligne], 91-2 | 2005, mis en ligne le 10 juin 2010, consulté le 03 septembre 2022. URL : http://journals.openedition.org/jsa/2953 ; DOI : https:// doi.org/10.4000/jsa.2953 


\title{
PROBLÈMES DE STRUCTURE DANS LES ANDES. DE LA PARENTÉ, DE LA POLYGYNIE ET DES MOITIÉS À CUZCO
}

\author{
R. Tom ZUIDEMA *
}

\begin{abstract}
Lévi-Strauss, dans des articles de 1983, pose le problème des choix multiples, avec des avantages différents, dans les relations de parenté unissant un chef et ses sujets. II prend pour premier exemple d'analyse la cour d'Heian dans le Japon du XI ${ }^{\mathrm{e}}$ siècle. Un exemple comparable avait été donné par van Wouden (1956) concernant la région de Kodi, dans l'île de Sumba (Indonésie) et Lévi-Strauss (1958 [1956]) lui-même avait auparavant traité le cas des Bororo. Sur trois des aspects de la question discutée par Lévi-Strauss on montrera leur importance dans l'empire inca à partir de cas concrets. [Mots clés: Incas, Cuzco, structuralisme, histoire, parenté, polygynie, organisation politique.]
\end{abstract}

Problems of structure in the Andes. Kinship, polygyny and moieties in Cuzco. LéviStrauss, in articles written in 1983, poses the problem of multiple choices, with different advantages, of kin relationships between a ruler and his subjects. He takes as a first example of analysis the Heian court in Japan of the eleventh century. A comparable example had been given by van Wouden in 1956 from the lanscape of Kodi on the island of Sumba, Indonesia, and Lévi-Strauss (1958 [1956]) himself had referred before to that of the Bororo, South America. Taking three aspects of the problem as discussed by Lévi-Strauss, I demonstrate the importance of these in the case of the Inca empire, Peru, and illustrate them with examples taken from here. [Key words : Incas, Cuzco, structuralism, history, kinship, polygyny, political organization.]

Problemas de estructuras en los Andes : parentesco, poliginia y mitades en Cuzco. En artículos de 1983, Lévi-Strauss plantea el problema de las opciones múltiples - con distintas ventajas - ligadas a las relaciones de parentesco entre un dirigente y sus sujetos. Como primer ejemplo analiza la corte de Heian en el Japón del siglo XI. Un ejemplo semejante estudiado por van Wouden (1956) concierne la región de Kodi en la isla de Sumba (Indonesia) y el mismo Lévi-Strauss (1958 [1956]) había tratado con

* University of Illinois, Department of Anthropology, 109 Davenport Hall, 607 South Mathews Avenue, Urbana, IL 61801, États-Unis [rtzuidem@uiuc.edu].

Journal de la Société des Américanistes, 2005, 91-2, pp. 31-49. (O) Société des Américanistes. 
anterioridad el caso de los bororos. Sobre tres aspectos del problema discutido por Lévi-Strauss, se mostrará aquí su importancia para el imperio inca, partiendo de datos concretos. [Palabras claves : incas, Cuzco, estructuralismo, historia, parentesco, poliginia, organización política.]

Outre l'importance de l'œuvre de Lévi-Strauss pour mon approche de l'anthropologie en général, des études plus spécialisées de ce dernier m’ont été précieuses pour comprendre la culture andine à l'époque de l'empire inca. Mais, en traitant de ce sujet, je me suis trouvé confronté, d'une part, à ma formation culturelle et intellectuelle issue de la littérature hollandaise sur l'Indonésie, d'autre part, au fait que toutes nos connaissances et toute notre sensibilité sur la culture inca sont passées par le filtre des écrits espagnols de l'époque coloniale, enfin à la culture nord-américaine dont j'utilise maintenant la langue pour parler des Incas. Même si mes préoccupations avaient un caractère purement anthropologique, à savoir celles d'un chercheur de terrain étudiant une culture étrangère, j'ai été amené à faire des recherches historiques non pas sur le passé inca, mais sur le passé colonial espagnol.

Ces circonstances ont fait que mon tout premier contact avec la culture inca eut lieu à travers l'organisation rituelle de Cuzco, la capitale inca, sujet sur lequel les Espagnols ont fait preuve d'ambivalence. D'une part, nous avons une description extrêmement formelle et conceptualisée de cette organisation grâce au système des ceques : l'abstraction même de ce dernier les incita à le décrire dans les moindres détails. On a pu vérifier empiriquement la fiabilité de ce système via l'aspect temporel de sa fonction sociale, à savoir le comput des jours de l'année solaire, dont les fondements astronomiques sont attestés par des vestiges archéologiques. D'autre part, certaines conséquences socio-politiques de cette organisation ont suscité l'intérêt intellectuel des Espagnols pour les débats juridiques et théologiques dans lesquels se trouvaient impliqués les descendants des Incas et leurs familles qui étaient obligés de défendre leurs droits de succession et leurs droits fonciers selon la loi espagnole. Au cours de ces débats étaient en effet commentées les anciennes façons de penser indigènes.

Après avoir analysé l'organisation de Cuzco dans le contexte du système des ceques (Zuidema 1964), j'entrepris de décrire la culture inca et, plus particulièrement, certains de ses aspects rapportés par les chroniques espagnoles et les documents coloniaux, tels que la parenté, l'organisation sociale, le calendrier, l'art, les mythes et les rituels. Mes questions se sont développées parallèlement à l'évolution des orientations théoriques de Lévi-Strauss, qui toutes étaient, d'une façon ou d'une autre, en relation avec l'importance théorique du mariage entre cousins croisés et son rôle dans la société. J'examinerai tout d'abord trois de ces orientations dans la mesure où elles m'ont aidé à aborder les questions que je me suis posées en ce qui concerne les Andes. Comme elles n'ont cessé de me 
préoccuper au long des années, je les illustrerai chacune par un exemple faisant appel à des données historiques plus précises. Ces trois exemples me permettront de clarifier et de faire progresser la discussion sur les conceptions incas relatives à la structure socio-politique, et de souligner à nouveau l'importance des hypothèses lévi-straussiennes.

La première question concerne l'équivalence terminologique de parenté entre le « frère de la mère » et le « frère ou père de la femme » en quechua et en aymara, les deux principales langues parlées dans l'empire inca. Je parvins tout d'abord à la conclusion que cette équivalence concernait le mariage avec la fille du frère de la mère et tentai, dans mon interprétation du système social inca, d'attribuer un rôle au « connubium asymétrique » entre groupes donneurs et groupes preneurs de femmes, théorie développée par l'anthropologie hollandaise pour les sociétés indonésiennes (Duyvendak 1926 ; Wouden 1968 [1935]) et reformulée par LéviStrauss (1949) sous le nom d'« échange généralisé » dans son livre Les Structures élémentaires de la parenté qui a considérablement élargi l'horizon théorique et géographique des problèmes tels qu'ils avaient été étudiés jusqu'alors ; le long et très élogieux compte rendu qu'en fit J. P. B. de Josselin de Jong (1952) faisant néanmoins ressortir une différence d'approche. Ainsi, tandis que Lévi-Strauss, selon Josselin de Jong, minimisait le rôle des structures " bilinéaires » (" bilatérales » dans la terminologie lévi-straussienne) ou de "double-descendance » dans les systèmes sociaux, Josselin de Jong insistait sur leur importance fondamentale, non seulement dans les systèmes australiens à échange symétrique entre les groupes, mais aussi dans les systèmes indonésiens à échange asymétrique. En Indonésie, ces structures permettaient en outre d'expliquer la distribution éparse ou la combinaison de traits patrilinéaires et matrilinéaires. Je ne mentionnerai pas ici les similarités frappantes que j'ai pu observer entre les systèmes sociaux indonésien et andin - s'agissant en particulier des unités politiques, de leurs sous-unités et des rôles rituels qui leur étaient attribués. Il est plus intéressant de noter que, dès le début, apparurent de sérieux problèmes en ce qui concerne l'application de la théorie du « connubium asymétrique » au contexte péruvien, problèmes en partie entrevus par van Wouden lorsqu'il tenta plus tard d'appliquer cette théorie au terrain (Wouden 1983 [1956]).

À Kodi, partie de l'île de Sumba en Indonésie de l'Est, van Wouden observa que les mariages entre cousins croisés obéissaient à des stratégies familiales particulières et, à un moindre degré, renouvelaient ou concluaient des alliances entre des groupes sociaux plus larges. Ainsi, une famille s'arrangeait pour qu'un de ses membres se marie avec la fille de la sœur du père, c'est-à-dire le type de mariage le plus endogamique, qu'un autre épouse la fille du frère de la mère, mariage moins endogamique, et que tous les autres trouvent un conjoint dans la parenté éloignée ou hors de la parenté. L'organisation sociale reflétait ces préférences de deux façons. D'une part, il existait des divisions territoriales patrilocales (quatre en principe, dont le rang dépendait des fonctions rituelles qu'elles rem- 
plissaient) qui comprenaient des villages et des maisons et montraient une tendance endogamique - notons cependant que si, dans l'idéal, les maisons étaient patrilinéaires, une personne pouvait changer d'appartenance en allant ailleurs. D'autre part, il y avait des "milliers ", mais en réalité seulement une soixantaine de clans matrilinéaires, non localisés et strictement exogamiques; celui qui naissait dans un clan appartenait toute sa vie à ce clan. Les clans étaient hiérarchisés par ordre décroissant en fonction de leurs origines plus ou moins lointaines par rapport à l'extérieur, ce qui réduisait ou augmentait leurs pouvoirs en matière de sorcellerie. Les familles organisaient leurs stratégies matrimoniales en combinant les différents avantages qu'elles avaient à leur disposition.

La deuxième question a été analysée par Lévi-Strauss (1958) dans son article intitulé « Les organisations dualistes existent-elles ? ». Cet article parut, en 1956, dans un volume d'hommages consacré à J. P. B. Josselin de Jong dans lequel figurait également celui de van Wouden. Avec le recul, ils apparaissent complémentaires. Après avoir rappelé les exemples indonésiens du problème qu'il pose, Lévi-Strauss décrit en détail l'exemple des moitiés winnebago en Amérique du Nord. Les informateurs de la moitié «d'en-Haut » situaient les moitiés en position diamétralement opposée l'une par rapport à l'autre à l'intérieur du village, alors que les informateurs de la moitié « d'en-Bas » disaient que les chefs de chacune des deux moitiés vivaient au centre du village et que les autres gens vivaient à la périphérie. C'est en étendant ce problème à des exemples sudaméricains que Lévi-Strauss a donné son analyse la plus technique d'un village bororo. Je me contenterai ici d'observer que, si les deux moitiés occupaient des demi-cercles égaux dans le cercle formé par le village, elles exprimaient aussi une opposition intérieur/extérieur qui se traduisait par la possibilité pour une moitié, celle à laquelle appartenait les deux chefs de village, de contracter certains mariages endogames qui étaient interdits dans l'autre.

C'est en réalisant les multiples possibilités qu'offrent certaines cultures qui combinent et/ou transforment l'une dans l'autre plusieurs formes d'organisation duelles - diamétrales ou concentriques - et triadiques, que je suis parvenu à décrire l'ordre social formel de Cuzco tel qu'il est codifié par le système des ceques. Au sein de chacune des quatre divisions spatiales (I, II, III, IV) sont définis neuf rangs (par ordre décroissant : 1 a, b, c ; 2 a, b, c ; 3 a, b, c). Les noms des catégories duelles et triadiques, ou leurs synonymes attestés, pouvaient, selon les circonstances, changer de position ou être ré-ordonnés. Pour m'en tenir à deux exemples, les noms des rangs a, b, c pouvaient s'appliquer aux groupes I, II, III, et les noms des moitiés I + III et II + IV être donnés à certaines relations au sein de la hiérarchie triadique. Pour ce deuxième exemple, je montrerai dans quel contexte légal les gens tiraient profit de ces transformations ou équivalences.

La troisième question soulevée par Lévi-Strauss (1982; 1983a ; 1983b ; 1983c) permet de situer les problèmes spécifiques de l'organisation de Cuzco dans une perspective théorique plus large, à savoir une comparaison avec certaines 
formes de royaume et de gouvernement, et la nécessité de combiner les approches anthropologique et historique. Ses deux premiers exemples concernent la cour japonaise de la période Heian ( $\mathrm{xl}^{\mathrm{c}}$ siècle) et le royaume Merina du centre de Madagascar ( $\mathrm{XIX}^{\mathrm{e}}$ siècle). Tous deux montrent que les gens étaient conscients des avantages que leur offrait telle ou telle forme plus ou moins endogamique ou exogamique de mariage ainsi que des changements de préférences matrimoniales au cours du temps. Les préoccupations de Lévi-Strauss rejoignent celles de Condominas à propos de Madagascar et celles de P. E. de Josselin de Jong à propos de l'Indonésie de l'Ouest, préoccupations qui apparaissent explicitement dans le titre de l'article de Condominas : «Le souverain époux de son peuple : variations madécasses sur un thème malais » (Josselin de Jong 1980 ; Jordaan et Josselin de Jong 1985 ; Condominas 1989). Les détails très techniques de ce texte fournissent à Lévi-Strauss la base de son argumentation. Dans le même volume que celui du texte de Condominas figurait un de mes articles où je tentais de définir une perspective andine quant à l'équivalence terminologique « frère de la mère/père de la femme » (Zuidema 1989). Dans mon dernier exemple, j'essaierai de montrer l'importance de cette équivalence pour les problèmes traités par Lévi-Strauss en 1983, et que van Wouden avait soulevés en 1956.

Avant d'en venir à mes trois exemples, examinons une question concernant l'histoire et la structure dans les Andes. Les chroniqueurs espagnols mentionnent certains traits de la culture andine, observés par eux-mêmes ou rapportés par des informateurs, qui, à l'évidence, avaient une grande importance, mais à propos desquels ils n'ont fourni que peu de détails. Nous mesurons cette importance lorsque nous découvrons des traits similaires décrits plus amplement au sujet d'autres cultures, anciennes ou récentes, de l'Amérique du Sud. Cela m'a toujours beaucoup aidé d'avoir présent à l'esprit l'ensemble de l'Amérique du Sud indigène en tant que « terrain d'étude ethnologique » (Josselin de Jong 1935; Urton 1996). Encore aujourd'hui, je suis émerveillé par la prescience de Lévi-Strauss (1958, pp. 102, 109) qui, en 1956, suggérait une similarité possible entre le village bororo, Tiahuanaco et Cuzco. Si j'ai évité au maximum d'utiliser des matériaux comparatifs lorsque je tentais de déchiffrer le sens des données incaïques, dans d'autres textes, j'ai accordé une attention toute spéciale aux parallèles structuraux avec des sociétés du Brésil central (Zuidema 1998; Fabian 1998) ou de l'Amazonie du Nord (Zuidema 2002). Étant donné l'ampleur des comparaisons structurales de Lévi-Strauss, il serait intéressant de déterminer dans quelle mesure ses critères se distinguent de ceux de l'école de Leyde (Josselin de Jong, éd., 1977 ; Rassers 1959 ; Ridder et Karremans 1987) ${ }^{1}$. Les exemples suivants s'inscrivent, à mon sens, au cœur de la structure socio-politique inca et de la vision qu'avaient les Incas de leur passé. Les problèmes qu'ils posent suscitèrent, au-delà de leurs intérêts politique et théologique, un intérêt culturel et des débats juridiques passionnés de la part des Espagnols. Il s'agit de problèmes structuraux qui nécessitent une analyse historique. 
Revenons maintenant à l'exemple andin susceptible d'éclairer notre première question. L'équivalence entre le frère de la mère et le frère ou le père de l'épouse s'éclaire lorsqu'on y voit la conséquence d'une autre équivalence, celle qui fait que, dans la hiérarchie inca, un homme, pour des raisons de succession, distingue entre deux fils en appelant le premier churi, "fils d'un homme ", et le second concha, " fils de la sœur d'un homme », et non en l'expliquant par le modèle de l'échange généralisé. Cette distinction suscita d'emblée l'intérêt de Juan de Betanzos (1987 [1551], chapitres XII, XIV, XVI), le premier chroniqueur à qui l'on doit une description exhaustive de la culture inca à Cuzco en 1551, dix-huit ans après l'arrivée des Espagnols au Pérou. Décrivant les pratiques relatives au mariage royal, il déclare tout d'abord que le roi (capac, « royal, riche »), la reine (cuya), ainsi que la haute noblesse étaient huaccha cuyac « ceux qui aiment les pauvres » (huaccha " pauvres, sans parenté », cuyac " celui/celle qui aime»). Une classe intermédiaire se composait des huaccha concha, terme qui, malgré son sens littéral de "fils de sœur pauvre », désignait les descendants des hommes de la famille royale mariés à des femmes non incas ${ }^{2}$. Betanzos explique la fonction de cette hiérarchie au sein de l'organisation politique (mais aussi économique et rituelle) de Cuzco et des alentours. Avant d'épouser la reine, le roi qui avait instauré cet ordre s'était assuré de l'amitié des chefs locaux des environs de Cuzco en offrant à chacun d'eux une femme " de son lignage » comme « épouse principale ». Il espérait ainsi obtenir l'allégeance de leurs fils en tant que successeurs de leurs pères. Puis, après avoir épousé la reine ainsi qu'une autre femme noble (chacune représentant probablement une moitié de la vallée de Cuzco), il reconnut leurs fils (la première en eut deux, la seconde, un seul) en célébrant leur naissance (Betanzos ibid., chapitre XX). Par ailleurs, dix chefs de haut rang et dix chefs de rang inférieur des environs de Cuzco l'acceptèrent comme seigneur auquel ils devraient rendre des services sous forme de travail et de qui ils obtiendraient des faveurs ; chacun d'eux lui offrit une sœur en mariage. Betanzos décrit comme historique une suite d'événements où un futur roi commence par distribuer ses sœurs en tant qu'épouses principales à des nobles qui ne sont pas de Cuzco et reçoit, une fois devenu roi, en tant qu'épouses secondaires des sœurs de ces mêmes nobles. Il ne nous donne pas plus de détails sur ce que devinrent les fils de ces femmes. Une histoire relatée par des chroniqueurs plus récents à propos d'un roi précédent nous fournit cependant une réponse partielle à cette question. C'est en tant que " fils de sœur » - avant qu'il ne soit couronné - que les membres du groupe de sa mère lui demandèrent d'être leur chef. D'autres données indiquent que cet épisode de l'histoire correspondait à une pratique très générale (Zuidema 1997).

Betanzos est explicite en ce qui concerne l'équivalence entre " fils secondaire » et "fils de sœur». Je commencerai donc par examiner ses répercussions sur d'autres équivalences de parenté, bien qu'il faille pour ce faire recourir à des 
sources plus récentes ${ }^{3}$. Les témoignages d'auteurs contemporains de Betanzos permettront de mieux comprendre l'intérêt que la société coloniale portait à ce problème.

Le frère cadet d'un homme s'adressait (ou était obligé de s'adresser) à son neveu, c'est-à-dire au fils de cet homme, non pas en utilisant le terme churi, " fils », mais le terme mulla, « fils de frère d'une femme »; le frère cadet transformait ainsi sa propre position en celle d'une sœur. $\mathrm{Si}$, donc, un frère plus âgé appelait un fils son "fils de sœur », non seulement il remplaçait une position d'épouse secondaire par celle de sœur, mais il se représentait le rôle intermédiaire de celle-ci comme celui d'un frère cadet. Dans ce contexte, l'explication donnée par Bertonio (1984 [1612]) en aymara pour l'équivalence entre le frère de la mère et le frère ou le père de l'épouse se comprend mieux s'agissant de la société andine. Bertonio dit que le terme utilisé par un homme pour désigner les parents masculins de sa femme ( $\mathrm{y}$ compris le frère et le père de celle-ci) était également utilisé par son fils pour désigner les mêmes personnes. Un parent affin du père devenait un frère de la mère pour le fils et entrait dans le cercle de famille de celui-ci, même s'il restait à la même distance qu'un " fils de sœur ». Dans le contexte des équivalences précédentes, je propose pour celle-ci l'interprétation suivante. Seul le fils qui succédait à son père, et plus généralement tout successeur d'un chef, pouvait l'appliquer. La succession donnait le droit d'obtenir plusieurs femmes des mains du souverain, d'hériter des femmes de son prédécesseur sous réserve d'observer une abstinence sexuelle avec ces dernières, enfin de se marier avec une parenté plus proche que celles permises aux hommes de rang inférieur. Un héritier succédait moins à son prédécesseur qu'il n'occupait la position que celui-ci avait laissée vacante. Toutefois, cette hypothèse n'a pu être entièrement vérifiée dans la mesure où nous manquons d'informations ethnohistoriques. Rien dans la terminologie de parenté inca n'indique qu'un choix était possible, comme dans la société andine actuelle, entre caca, «frère de la mère, père de la femme ", et suegro, terme espagnol utilisé uniquement pour les affins. En revanche, on est certain que l'équivalence inca pour caca n'impliquait aucune forme d'alliance asymétrique ou d'échange généralisé. Aucune autre équivalence ne pouvait étayer un tel système. Les trois équivalences dont nous venons de parler étaient la conséquence de choix faits par ou pour un individu et entraînaient des distinctions de rang au sein d'un groupe. L'équivalence entre le frère de la mère et le père de l'épouse avait probablement pour conséquence que celui qui s'en prévalait pouvait se marier dans une parenté plus proche.

Intéressons-nous maintenant aux données que nous ont laissées trois contemporains de Betanzos. Bien qu'elles impliquent toutes l'équivalence entre fils secondaire et fils de sœur dans des contextes similaires, chacun de ces auteurs en souligne un aspect différent. Cieza de Léon avait séjourné à Cuzco en $1550 \mathrm{au}$ moment où Betanzos y écrivait sa chronique, mais sa description générale de l'histoire et de la culture incas montre un savoir moins exhaustif du contexte local 
que chez ce dernier (Cieza de León 1986, I). Agustín de Zárate (1995 [1555]) est l'auteur de la première histoire de la conquête espagnole du Pérou, laquelle comporte plusieurs chapitres sur les Incas, et il a vraisemblablement connu Betanzos à Cuzco environ cinq ans avant Cieza de León. López de Gómara (1965 [1552]) a publié une histoire de la découverte de l'Amérique par les Espagnols qui comporte, elle aussi, plusieurs chapitres sur les Incas, mais il n'a jamais quitté l'Espagne. Alors que le livre de Betanzos ne fut publié que récemment, les ouvrages de Cieza de León, de Zárate et de López de Gómara parurent en 1554-1555 à Anvers. Dans quelle mesure ces derniers ou leurs éditeurs ont-ils eu connaissance de leurs ouvrages respectifs ? Enfin, tandis que trois de ces auteurs ont recueilli leurs informations dans le contexte cuzquénien, un seul, Cieza de León, note que les siennes proviennent de ce qui est aujourd'hui la Colombie et qui, à l'époque, ne faisait pas partie de l'empire inca. Tous, à l'exception de Betanzos, livrent aussi des informations sur certaines pratiques entourant les funérailles des nobles et des membres de leurs familles. On peut se demander dans quelle mesure ils se sont plus intéressés à la signification structurale des données qu'à leur situation géographique.

López de Gómara (1965, p. 219) retient du gouvernement de Cuzco la caractéristique suivante : « c'est le neveu qui hérite, non les fils ; seuls les Incas héritent de leur père par droit d'aînesse ». Apparemment, il déduisit de ses sources que c'était uniquement à l'occasion d'une succession royale qu'un fils pouvait hériter, mais que, dans tous les autres cas, c'était un concha. López de Gómara n'a pas vu que, dans ce contexte, le terme inca ne signifie pas tant « fils de sœur » que « fils secondaire » ou « fils cadet ». En revanche, Zárate (1995, chapitre XIII, p. 56) mentionne qu'à l'époque où les Incas de Cuzco conquirent tous les territoires et firent des tributaires, la coutume voulait que, chez eux et chez les natifs, la succession n'aille pas directement aux fils, «mais d'abord au frère du défunt né immédiatement après lui, et à la mort de celui-ci [que] le titre retourne au fils aîné de son frère [...] et [que] les choses se passaient toujours de cette façon ». En d'autres termes, le frère et le fils du roi régnant avaient tous deux le rang de « fils de sœur » jusqu'à ce qu'ils soient en position de successeurs ; et la succession au rang comme au titre était dictée par l'âge relatif et non par l'appartenance à une génération. Cieza de León comprend le modèle de succession qu'il a observé dans plusieurs chefferies de Colombie de la même manière que López de Gómara pour Cuzco, avec de légères variations dues au contexte local. Il répète à plusieurs reprises qu'en l'absence d'un fils, un fils de sœur était préféré à un frère. Mais cette insistance traduit une certaine gêne et on peut penser qu'il a confondu une équivalence avec un choix occasionnel. Dans un autre passage de son livre, il décrit les choses d'une façon différente qui correspond probablemement à ce qui se passait dans tous les cas. Il raconte qu'on déposait les nobles de haut rang dans des tombes très grandes et très profondes en compagnie de plusieurs de leurs épouses et servantes. Les nobles du voisinage offraient deux ou trois hommes et 
des femmes (en tant qu'épouses du défunt ?) et les déposaient dans la tombe de ce dernier afin de lui tenir compagnie. Ces nobles défunts n'avaient pas moins de vingt personnes auprès d'eux dans leur sépulture. Cieza de León apporte ici la preuve la plus convaincante que la polygynie et l'accompagnement dans la tombe traduisaient un système de domination par le biais des mariages secondaires pendant la vie et/ou la mort. De plus, les funérailles offraient aussi l'occasion d'exercer de l'extérieur, avec une certaine efficacité, des pressions politiques sur l'autorité centrale, ou celle momentanément reconnue comme telle. C'est lors de ce moment exceptionnel qu'un nouveau chef devait être choisi.

À l'époque où ces auteurs décrivaient les divers aspects de ce système social, les structures indigènes de celui-ci dominaient encore leur façon de l'appréhender: Mais les germes des interprétations futures étaient déjà présents. La preuve en est le remplacement, dans une nouvelle édition de la chronique de Zárate datant de 1577, de la phrase qui décrit la succession d'un frère ou d'un fils. Entre-temps, la succession du frère avait été interdite par la loi, le gouvernement colonial ayant réalisé son importance pour les factions au sein des familles nobles polygynes (Ragon 1992). En réorganisant l'administration coloniale à partir de 1569, le nouveau vice-roi Francisco de Toledo défendait la légitimité de la présence du gouvernement royal espagnol et condamnait l'ancienne « tyrannie » dont avaient fait preuve les rois incas en conquérant le pays. Dans les chroniques à partir d'environ 1572, lesdits événements conduisirent à cette curieuse conclusion que la succession royale inca avait toujours été celle du père au fils de la reine et que la conquête de l'empire en était le résultat. Dans la chronique de Zárate, la phrase se trouve modifiée, sans doute en raison de pressions politiques, de la façon suivante : «à partir de ce moment-là, celui qui détenait le pouvoir et la force devint le chef sans que fût respecté l'ordre de succession légitime et au moyen de la violence et de la tyrannie... " (Duviols 1964).

L'exemple qui illustre la seconde question, celle relative à l'existence de perspectives différentes sur l'organisation dualiste de Cuzco, représente peut-être la réaction la plus consciente à des revendications différentes et changeantes concernant la succession inca, bien qu'il s'agisse d'un litige juridique portant sur des droits fonciers à l'époque coloniale (1560). Celui-ci a pour contexte l'administration inca de la vallée de Cuzco, laquelle n'avait pas encore perdu de son influence. La vallée avait été divisée en dix unités locales hiérarchisées : cinq dans la moitié hanan "d'en-Haut» et cinq dans la moitié hurin "d'en-Bas ». Le gouvernement de l'unité de plus haut rang avait été attribué par un précédent roi à son " fils », churi. Celui-ci allait être le premier roi qui épouserait sa vraie sœur. Les neuf autres unités furent attribuées à autant de « fils de sœur », concha, dont les rangs furent distingués les uns des autres en fonction de leur distance généa- 
logique supposée avec le roi. Ainsi le passé était interprété à partir du « présent ethnographique » du règne de ce roi considéré comme l'instaurateur de ce type d'administration. Il n'était pas présenté comme le résultat du processus historique progressif d'une dynastie royale, à l'instar de celui qui, dans l'esprit des Espagnols, conduisit plus tard à la nécessaire introduction du seul et véritable dieu, à savoir le Dieu chrétien.

Le litige, qui portait sur un terrain situé dans la partie hurin de la vallée, opposait un homme qui revendiquait son appartenance à la quatrième panaca (groupe de descendance) de la moitié hanan et se prétendait être le descendant noble du quatrième souverain de cette moitié, et un homme qui avait la même revendication en ce qui concerne la quatrième unité et le quatrième souverain de la moitié hurin. La décision finale fut prise en 1560 par le juriste Polo de Ondegardo, alors gouverneur de Cuzco et l'auteur le plus célèbre qui écrivit sur les Incas (Sherbondy 1996). Il attribua la terre en question au noble hurin, arguant qu'il était bien connu, selon la loi inca, que les gens d'une moitié ne pouvaient pas posséder de terre dans l'autre moitié. Pour nous, il est du plus haut intérêt de savoir comment le noble hurin défendit sa cause sur le plan généalogique, et comment, par la suite, hors du contexte de ce document juridique mais dans un de ses écrits sur la religion inca, Polo de Ondegardo inscrivit cette généalogie dans une description de la dynastie inca.

Don Juan Tambo Uscamayta prétendait être l'arrière-petit-fils de Mayta Capac, quatrième roi de hurin et chef de la panaca Usca Mayta. Son grand-père, Tarco Huaman, fils de Mayta Capac, avait vécu à l'époque des Incas, et son père, Don Antonio Quispe Uscamayta, avait été témoin de la conquête de l'empire par les Espagnols. Sa lignée remontait au fondateur de sa panaca et était aussi longue que celle de son adversaire de la moitié hanan. En 1560, était encore répandue l'idée que les rangs des panaca équivalentes de hanan et de hurin étaient liés les uns aux autres par un même ancêtre au sein d'un système de cinq générations, de même que l'idée qu'une dynastie de cinq rois hurin avait précédé une dynastie de cinq rois hanan. Le onzième roi, celui qui acheva la conquête de l'empire inca, n'était plus représenté par une panaca supplémentaire dans la vallée de Cuzco. Sous son règne, les Espagnols avaient déjà exploré les côtes nord de l'empire et ce roi fut le dernier qui mourut selon les traditions incas.

On comprend pourquoi Don Juan défendit une lignée courte qui le rattachait à l'origine juridique de sa panaca. Quelle que soit l'idée qu'il se faisait de ce que les Espagnols savaient ou non de la dynastie inca, cette stratégie était plus avantageuse pour lui que de prétendre être le descendant d'un roi distant de cinq générations de plus, ce qui lui aurait donné des droits moins évidents sur les terres de sa panaca. Aucune autre revendication de son ascendance royale ne figure dans le document. En procédant de la même façon, plus tard, hors du contexte du litige, Polo de Ondegardo mêle une conclusion logique à une nouvelle et curieuse distorsion dans sa façon de présenter les faits. Il mentionne d'abord hanan, la 
moitié " principale », comme la seule gouvernant l'ensemble de Cuzco, ainsi que sa dynastie telle qu'elle était généralement connue, et seulement ensuite la moitié hurin. Mais ici, après avoir énuméré les rois jusqu'à Mayta Capac, il passe tout d'un coup à la lignée secondaire de la panaca privée de celui-ci comme si elle faisait partie de la même lignée royale ! Le nom du cinquième roi est placé en position inférieure dans la lignée royale deux générations avant Mayta Capac.

Polo de Ondegardo était parfaitement conscient du caractère mythique, rituel et calendaire de l'organisation qu'il décrivait. Il évoque d'abord les « statues ou les pierres » qui représentaient les ancêtres et les identifie grâce aux noms de ces derniers. L'ancêtre commun des deux lignages était sorti d'une cave après le Déluge ; les « idoles » étaient portées en procession à l'occasion de leurs rituels calendaires. Polo de Ondegardo était également conscient d'un problème similaire à celui que pose Lévi-Strauss dans son article « Les organisations dualistes existent-elles ? ». Bien qu'en positions diamétralement opposées dans la vallée, les deux groupes de panaca, hanan et hurin, étaient censés (selon d'autres auteurs contemporains) être liés l'un à l'autre en tant que respectivement « fils » et « fils de sœur ", le premier ayant des mères issues de l'intérieur de la vallée, le second ayant des mères venant de l'extérieur de cette même vallée. Cependant, si l'on ne considère qu'une seule dynastie, la partie hurin était le " père » et la partie hanan le « fils ». Les deux façons concurrentes de se représenter la hiérarchie des panaca renvoyaient au passé. Il est certain que, pour diverses raisons de nature politique et selon les intérêts divergents des deux moitiés, les Incas pouvaient recourir, soit à l'idée d'une seule lignée royale, soit à celle d'une lignée de "fils de sœur " existant avec une lignée de " fils ». Cependant, quand, en 1572, une seule dynastie avec un seul type de succession, celle du père au fils, devint un dogme, son origine juridique, à savoir le système des dix territoires panaca, cessa, et pour cause, d'être invoquée.

Venons-en maintenant au troisième exemple qui illustre l'importance des articles que Lévi-Strauss publia en 1983. Plusieurs chroniqueurs assignent un rôle précis à la polygynie et un rôle potentiel et secondaire à une certaine forme de polyandrie. Le fondateur légendaire de ce système était censé avoir engendré cent fils et cinquante filles; ces " bâtards » constituaient un hatun aillo, ce qui signifie " grand lignage ». Celui-ci était également appelé iñaca panaca (c'est-à-dire le groupe de descendance des sœurs pana, celles-ci étant les dames non incas iñaca). Ce roi « prit toutes ses sœurs pour maîtresses, prétendant qu'elles ne pouvaient avoir de meilleur mari que leur frère " (Sarmiento de Gamboa 1942 [1572], chapitre XLVII). (On ne peut trouver plus juste description du roi comme preneur de femmes et donneur de femmes à ses sujets, c'est-à-dire comme "souverain époux de son peuple ", pour reprendre l'expression de Condominas.) La même 
stratégie de mariage était utilisée par les autres chefs. Offĭciellement, quand un homme accédait à une position plus élevée, son nouveau rang se définissait par le droit d'avoir un plus grand nombre d'épouses qu'auparavant, par le rang d'une nouvelle épouse principale et par le privilège consistant à se marier avec une parente toujours plus proche, allant d'une non-cousine à une cousine, puis à une demi-sœur, enfin, dans le cas du roi, à une vraie sœur. Avoir un plus grand nombre de femmes voulait dire avoir plus d'enfants et pendant une plus longue période, ce qui étendait une génération dans le temps et augmentait les possibilités de succession en faveur des frères. Les descendants nobles des anciens rois incas étaient mariés de la main même du roi, mais les nobles non incas et les femmes proches de la cour - y compris les dames iñaca - l'étaient seulement en présence du roi et non de ses mains. Comme ces femmes étaient aussi considérées comme des ancêtres des lignées secondaires, à savoir les panacas constituées de « fils de sœur », on peut supposer qu'au moment où elles donnaient des fils à leurs maris, l'Inca engendrait en elles des " fils de sæur ». Un homme qui assumait à la fois les deux rôles, celui de " fils » et celui de " fils de sœur », pouvait peut-être succéder à son père « local » et être un délégué du roi.

Le rang d'une personne (homme ou femme), sa proximité par rapport au roi ou à la reine et son appartenance à telle ou telle panaca dépendaient non seulement du rang des deux parents, mais aussi de circonstances personnelles : le moment ou le mois de sa naissance, sa condition physique, sa bravoure lors de l'initiation ou à la guerre, ses aptitudes personnelles. Ainsi, par exemple, les conditions requises pour devenir prêtre « du soleil » - appelé tarpuntay " planteur » quand il effectuait des rituels au moment du cycle agricole - étaient d'abord d'avoir été conçu ou d'être né sous l'influence du tonnerre, ensuite de posséder les capacités requises et d'avoir été initié à cette profession. C'est seulement à un âge avancé qu'il pouvait devenir membre de la panaca à laquelle appartenaient ces prêtres. Nous ne possédons que de rares exemples de généalogies et d'histoires de vie où tel ou tel de ces facteurs est mentionné et joue un rôle. Ils peuvent avoir été choisis pour rapporter un événement historique ou illustrer un rituel en tant que partie de l'ordre cosmique; il se peut aussi que deux chroniqueurs aient utilisé la même information dans des circonstances très différentes. Illustrons cet exemple par l'histoire exceptionnelle d'une de ces lignées dont l'épisode final met en scène la princesse royale qu'épousa Betanzos - lequel a peut-être présenté les faits de son point de vue à elle. Quoi qu'il en soit, il s'agit de la meilleure description d'un type de pratique également rapporté par d'autres chroniqueurs, mais avec moins de détails.

Comme on l'a dit plus haut, Betanzos a décrit le système des alliances royales dans la vallée de Cuzco et ses alentours du point de vue du neuvième roi, Pachacuti Inca. Ce roi commença son règne comme prince rebelle avant d'être couronné. Cela peut expliquer que son successeur ait été nommé chef de la première des cinq panacas de la moitié hanan de Cuzco - on dit aussi de lui qu'il 
fut le premier roi qui épousa sa vraie sœur -, alors que son père resta dans la seconde panaca appelée iñaca. Pachacuti Inca reconnut officiellement deux des fils que lui avait donnés la reine. L’ainé, Yamqui Yupanqui, dirigea les premières grandes conquêtes vers le nord, la partie du futur empire qui aura le rang le plus élevé. Ces événements eurent lieu au moment où son père, alors âgé de 70 ans, institua les lois du royaume comme s'il allait bientôt mourir (Betanzos 1987, chapitre XXIII). Yamqui Yupanqui hérita de la couronne et régna sur un empire de plus en plus étendu alors même que son père était encore en vie (ibid., chapitre XXV). Pachacuti Inca souhaitait que le fils de Yamqui Yupanqui, qui portait le même nom que son père, soit désigné comme le prochain successeur ; mais, comme celui-ci était encore un enfant, son père donna la préférence à son jeune frère Tupa Yupanqui, auquel il fit épouser une jeune sœur de sa propre épouse (ibid., chapitre XXVI). Tupa Yupanqui fut donc couronné, et lorsque la reine mit au monde un fils, Pachacuti Inca demanda à Yamqui Yupanqui, le père de ce nom, de couronner cet enfant, nommé Huayna Capac, le « jeune roi », comme futur successeur. C'est seulement alors que Pachacuti Inca mourut à l'âge de 120 ans. Le rôle prédominant qu'avait d'abord joué le premier Yamqui Yupanqui en tant que conquérant et roi montrant un intérêt particulier pour l'agriculture dans l'empire (ibid., chapitres XXIV et XXVI), allait être repris, aux côtés de Huayna Capac devenu empereur, par le jeune Yamqui Yupanqui ; celui-ci se vit confier à son tour l'administration de tous les troupeaux de lamas de l'empire, ce qui était une fonction très importante, comme l'attestent également d'autres sources.

Cependant, le cœur de l'argumentation de Betanzos se situe à la génération suivante, celle des fils de Huayna Capac, Huascar et Atahuallpa. Le centre d'intérêt de l'empire inca s'était déplacé vers le nord, et, depuis Quito, Atahuallpa se mit à contester la position impériale de Huascar à Cuzco. Huascar et Atahuallpa étaient demi-frères et, tandis que Huascar était probablement de rang plus élevé, sa mère provenait de la partie hurin de la vallée et Atahuallpa appartenait à la panaca de Pachacuti Inca dans la partie hanan. Cusi Yupanqui, fils du jeune Yamqui Yupanqui, était le principal conseiller de Huascar. Mais quand celui-ci l'envoya comme médiateur auprès de son frère, Cusi Yupanqui changea de camp, revirement qui joua peut-être un rôle décisif dans la défaite infligée à Huascar par Atahuallpa. La conquête espagnole, avec l'épisode crucial au cours duquel Francisco Pizarro fit Atahuallpa prisonnier, mit fin aux développements incaïques, mais non aux développements coloniaux de la lignée de Cusi Yupanqui. La fille de celui-ci avait été désignée comme devant être l'épouse d'Atahuallpa, mais ils ne furent ni mariés ni couronnés. Ensuite, les jeux de pouvoir firent qu'elle devint la maîtresse de Francisco Pizarro, peut-être avec le consentement de son frère, et qu'elle épousa plus tard Betanzos. Atahuallpa avait éliminé une grande partie de la haute noblesse de Cuzco. Il voulait appeler sa nouvelle panaca Capac Ticci «commencement royal», entamer une nouvelle 
histoire et réécrire l'ancienne. Mais peut-être ces idées émanaient-elles déjà du Quito colonial (Hyland 2002, p. 156, note 6; Pachacuti Yamqui Salcamaygua 1993, f. 42).

La lignée de Yamqui Yupanqui, en tant que " fils de sœur », devait rendre une fille en tant que reine à Atahuallpa, confirmant ainsi la lignée royale de ce dernier. En ce sens, elle allait épouser un « fils de sœur de père » et lui une « fille de frère de la mère ». Cette façon de considérer ce mariage ne présuppose pas un modèle d'échange généralisé, mais doit être replacée dans le contexte des échanges matrimoniaux entre une famille royale et un grand nombre de familles qui lui apportaient leur soutien. Apparemment, Cusi Yupanqui avait le pouvoir d'obliger sa sœur à épouser Atahuallpa et à devenir reine. Cette forme de mariage a pu être interprétée comme s'inscrivant dans le processus historique suivant: 1) Pachacuti Inca conclut de multiples alliances avec ses sujets nobles par le biais de mariages secondaires ; 2) Tupa Yupanqui est le premier roi qui a épousé sa vraie sœur ; 3) Huayna Capac est le premier à être considéré comme l'empereur d'un État qui ne dépend plus du soutien des panaca au roi. Mais on peut également considérer que la logique inhérente à un tel système hiérarchique trouve son explication dans un processus historique qui s'est développé sur plusieurs générations.

Une des difficultés que l'on rencontre lorsqu'on veut tirer des conclusions à propos de l'État inca vient du fait que toutes les données dont on dispose proviennent d'une culture que les chroniqueurs n'ont jamais pu voir fonctionner comme un tout organique. Toutefois, il est possible que les représentants de l'État inca aient communiqué aux Espagnols des informations exactes sur son fonctionnement grâce aux éléments livrés par le système cosmologique, en particulier le calendrier. Bien que les équivalences terminologiques dont nous avons parlé donnent une idée assez précise du système politique dans son ensemble, on ne dispose de pratiquement aucune information laissant penser qu'il existait une pratique de renouvellement des alliances entre les familles ou des unités sociales plus larges. Betanzos et d'autres chroniqueurs se bornent à mentionner la façon dont chaque unité de temps dans le calendrier de Cuzco était reconnue lors d'un service rituel effectué, en présence du roi, par une femme représentant un groupe particulier. Vus sous cet angle, d'autres détails de la politique matrimoniale s'éclairent : par exemple, lorsque Cieza de León parle de tel défunt noble auquel des nobles du voisinage offraient des hommes et des femmes pour lui tenir compagnie dans sa tombe; ou lorsque Betanzos, décrivant les préparatifs des funérailles de Pachacuti Inca (chapitre XXIII), raconte que ce roi reçut 200 $(2 \times 100)$ fils et $100(2 \times 50)$ filles de ses épouses secondaires, et qu'avant sa mort (Betanzos 1987, chapitre XXIV) il donna toutes ses filles à des nobles locaux. Le 
roi gouvernait au moyen d'un système de distribution des mariages à tous ses sujets nobles. En fait, les nombres 100 et 50 jouaient également un rôle important dans la distribution calendaire des serviteurs royaux et des épouses secondaires ${ }^{4}$. L'histoire du lignage de Yamqui Yupanqui n'est qu'un exemple, mais sans doute encore représentatif de ces alliances politiques. La succession des frères faisait partie de la même stratégie royale, et l'âge de Pachacuti Inca lorsqu'il mourut, à savoir 120 ans, c'est-à-dire le double de l'âge normal, peut être interprété comme une illustration légendaire de cette stratégie.

Pour conclure, revenons au document signé par Polo de Ondegardo en 1560. Peut-être est-ce ce document qui illustre le mieux comment seule une compréhension à la fois structurale et historique des différents aspects de la culture inca peut faire avancer l'étude de celle-ci. Il est clair que Polo de Ondegardo accepte les motivations politiques personnelles du noble hurin, et qu'il en déduit, sur le plan politique, l'existence de lignées de succession parallèles dans les deux moitiés. Reste l'énigmatique question de savoir pourquoi un noble hanan ne pouvait pas posséder de terre dans la moitié hurin et vice versa. Betanzos est le seul chroniqueur qui nous livre des informations sur les formes d'alliance matrimoniale entre les groupes lorsqu'il raconte comment Pachacuti Inca institua une exogamie de moitiés à Cuzco et dans les villes et villages alentours. Cette mesure n'était-elle pas suffisante pour permettre à une épouse d'apporter des terres de l'autre moitié à son mariage? Aujourd'hui, au contraire, l'endogamie de moitié est un idéal partagé dans les pays andins - allant parfois jusqu'à considérer l'autre moitié comme un territoire interdit. Rien ne permet de penser que nous avons à faire à une coutume apparue récemment. L'interdiction de posséder des terres dans l'autre moitié s'accorderait mieux avec l'existence d'une règle endogamique plus ancienne. Les solutions proposées par Lévi-Strauss aux problèmes théoriques que pose l'exemple des sociétés winnebago et bororo pourront peut-être permettre d'élucider le problème inca que nous venons d'exposer. De même que les moitiés et leurs panaca correspondaient à des différences de rang chez les « fils » et les «fils de sœur» dans la société de Cuzco; de même chaque rang était représenté par une terre située dans un endroit différent. La haute noblesse réservait les mariages principaux à la moitié hanan et acceptait que des mariages secondaires soient conclus dans l'autre moitié. Dans ce contexte, les règles endogamiques concernant l'héritage foncier ont peut-être joué un rôle.

À première vue, la société et la culture incas n'ont pas grand-chose à voir avec la société et la culture de Kodi, en Indonésie, ni avec l'ancienne cour impériale du Japon. Toutefois, alors qu'à Kodi et à Cuzco les divisions politiques étaient pensées en termes patrilinéaires, dans ces deux sociétés les différences de rang les plus subtiles étaient définies par les groupes matrilinéaires, que ces derniers aient été réels ou reconnus comme tels par le choix et le subterfuge d'une équivalence terminologique. Au Japon, Lévi-Strauss note que fut toujours pratiqué un choix entre les avantages respectifs de formes de mariage plus ou moins endo- ou 
exogamiques, combinés avec des changements de préférences matrimoniales. Au Pérou, l'institution politique de la polygynie montre bien qu'un leader politique avait intérêt à combiner plusieurs formes de mariage. Un roi ou un noble pouvait en choisir une ou plusieurs, mais il se peut aussi que, comme à Kodi, les choix aient été répartis entre les frères d'une même famille. Quand Lévi-Strauss raconte comment, au Japon, certaines familles puissantes avaient continué à imposer à leurs filles un mariage avec l'empereur, on peut penser que quelque chose de semblable a pu exister dans l'empire inca s'agissant des relations entre la lignée de Yamqui Yupanqui et celle du roi. Il est probable que le système de gouvernement péruvien est devenu plus complexe au fur et à mesure que l'empire se développait et que ses dirigeants se montraient plus capables d'exprimer les valeurs qui le fondaient. Mais, en fin de compte, nous ne pouvons le savoir qu'à travers les pressions qu'exercèrent sur ce système les nouveaux souverains espagnols. *

* Manuscrit reçu en mai 2005, accepté pour publication en octobre 2005.

\section{NOTES}

* Texte traduit par Marie-Claire Beauregardt avec le concours de Nathan Wachtel.

1. Dans la mesure où l'étude de l'Indonésie en tant que " terrain ethnologique » a été réalisée selon la méthode structuraliste, les travaux de Rassers (1959 [1925, 1926, 1931, 1940]) sur les origines austronésiennes de la culture hindu-javanaise constituent une source plus ancienne.

2. Un concept similaire à celui de huacha cuyac, " qui aime les pauvres », se retrouve dans l'Église chrétienne primitive (Brown 2002).

3. Ayant traité de ces équivalences ailleurs (Zuidema 1977 ; 1989), je ne mentionnerai ici que ce qui est nécessaire à mon propos.

4. J'ai déjà fait des observations sur l'importance du calendrier pour le mariage et l'organisation socio-politique dans mon livre de 1986. Je les complète grâce à une étude plus large sur la conception du temps, du passé et du calendrier par les Incas dans un texte à paraître (s. d.).

\section{RÉFÉRENCES}

BERTONIO Ludovico

1984 Vocabulario de la lengua aymara, Xavier Albo et Felix Layme, éds, CERES, Cochabamba [1612].

Betanzos Juan de

1987 Suma y narración de los incas María del Cármen Martín Rubio, éd., Atlas, Madrid [1551].

Brown Peter

2002 Poverty and leadership in the later Roman Empire, University Press of New England, Hanovre, coll. " The Menahem Stern Jerusalem Lectures ». 
Cieza de León Pedro

1986 Crónica del Perú. Primera parte, Miguel Maticorena Estrada, éd., Pontificia Universidad Católica del Perú, Academia nacional de la historia, Lima.

Condominas Georges

1989 «Le souverain époux de son peuple : variations madégasses sur un thème malais ", in Henry J. M. Claessen, éd., Variant views : five lectures from the perspective of the "Leiden tradition » in cultural anthropology, Universiteit van Leiden, Faculteit der Sociale Wetenschappen, Vakgroep Culturele Anthropologie en Sociologie der Niet-Westerse Samenlevingen, Leiden, pp. 1-55, coll. « ICA Leiden » 84.

Duviols Pierre

1964 «La historia del descubrimiento y de la conquista del Perú de Augustín de Zárate, remaniée conformément aux vues historico-politiques du vice-roi Toledo », Études Latino-Américaines, 2, pp. 147-151, Faculté des Lettres et Sciences humaines, Aix-en-Provence.

DUYvendak Johan Philip

1926 Het Kakean genootschap van Seran, W. Hilarius, Almelo.

FABIAN Stephen M.

1998 "Waiting to tie the knot: thoughts on structural similarities between Bororo and Inca », Journal of the Steward Anthropological Society, 26 (1-2), pp. 19-36.

HYLAND Sabine P.

2002 "The royal khipu of Blas Valera », in Jeffirey Quilter et Gary Urton, éds, Narrative threads. Accounting and recounting in Andean khipu, University of Texas Press, Austin, pp. 151-170.

JordaAan Roy E. et Patrick Edward de Josselin de Jong

1985 "Sickness as a metaphor in Indonesian political myths », Bijdragen tot de Taal-, Land- en Volkenkunde, 141 (2-3), pp. 253-274.

Josselin de Jong Jan Petrus Benjamin de

1935 De Maleische archipel als ethnologisch studieveld, J. Ginsberg, Leiden.

1952 Lévi-Straus's theory on kinship and marriage, E. J. Brill, Leiden, coll. « Mededelingen van het Rijkmuseum voor Volkenkunde » 10.

Josselin de Jong Patrick Edward de

1980 Ruler and realm : political myths in Western Indonesia, Noord-Hollandsche uitgevers maatschappij, Amsterdam, Oxford, New York, coll. « Mededelingen der Koninklijke Nederlandse Akademie van Wetenschappen, Afd. Letterkunde » 43 .

Josselin de JoNG Patrick Edward de, éd.

1977 Structural anthropology in the Netherlands, Martinus Nijhoff, La Haye.

LÉvi-Strauss Claude

1949 Les structures élémentaires de la parenté, Presses universitaires de France, Paris. 
1958 "Les organisations dualistes existent-elles? », in Claude Lévi-Strauss, Anthropologie structurale, Plon, Paris, pp. 147-182 [première édition : 1956, Bijdragen tot de Taal-, Land- en Volkenkunde, 112, pp. 99-128, volume

1982 «Chanson madégasse », in Orients : pour Georges Condominas, Sudestasie, Paris/Privat, Toulouse, pp. 195-203.

1983a «Du mariage dans un degré rapproché », in Le regard éloigné, Plon, Paris, pp. 127-142.

1983 « "Histoire et ethnologie », Annales. Économies Sociétés Civilisations, 38 (6), pp. 1217-1231.

1983c «Lectures croisées », in Le regard éloigné, Plon, Paris, pp. 107-126.

LÓPEZ DE Gómara Francisco

1965 Historia general de las Indias, Iberia, Barcelona [1552].

Pachacuti Yamoui Salcamaygua Joan de Santa Cruz

1993 Relación de antigüedades deste Reyno del Pirú. Estudio etnohistórico y linguístico édité par Pierre Duviols et César Itier, Institut français d'études andines, Centro de estudios regionales andinos, Lima/Cuzco.

RAGON Pierre

1992 Les Indiens de la Découverte. Évangélisation, mariage et sexualité. Mexique, $X V I^{e}$ siècle, Éditions L'Harmattan, Paris.

RASSERS Willem Huibert

1959 Pañji, the culture hero. A structural study of religion in Java, Martinus Nijhoff, La Haye.

Ridder R. de et J. A. J. Karremans, éds

1987 The Leiden tradition in structural anthropology. Essays in honour of $P$. E. de Josselin de Jong, E. J. Brill, Leiden/New York.

Sarmiento de Gamboa Pedro

1942 Historia de los incas, Angel Rosenblatt, éd., Émécé editores, Buenos Aires [1572].

SHERBONDY Jeannette E.

1996 "Panaca lands : re-invented communities », Journal of the Steward Anthropological Society, 24 (1-2), pp. 172-202.

\section{URTON Gary}

1996 « R. Tom Zuidema, Dutch structuralism, and the application of the "Leiden orientation" to Andean studies ", in Gary Urton, éd., Structure, knowledge and representation in the Andes: studies presented to Reiner Tom Zuidema on the occasion of his 70th birthday, Journal of the Steward Anthropological Society, 24 (1-2), pp. 1-36, Urbana.

Wouden Francicus Antonius Evert van

1968 Types of social structure in eastern Indonesia, Martinus Nijhoff, La Haye [republication de la thèse doctorale Sociale structuurtypen in de Groote Oost, J. Ginsberg, Leiden, 1935]. 
1983 "Local groups and double descent in Kodi, West Sumba », in P. E. de Josselin de Jong, éd., Structural anthropology in the Netherlands, Martinus Nijhoff, La Haye, pp. 184-222 [première édition : 1956 sous le titre « Locale groepen en dubbele afstamming in Kodi, West Sumba ", Bijdragen tot de Taal-, Land-en Volkenkunde, 112, pp. 204-246, volume d'hommages à Jan Petrus Benjamin de Josselin de Jong].

Zárate Agustín de

1995 Historia del descubrimiento y conquista del Perú, Franklin Pease G.Y. et Teodoro Hampe Martínez, éds, Pontificia Universidad Católica del Perú, Fondo Editorial, Lima [1555].

Zuidema R. Tom

1964 The ceque system of Cuzco : the social organization of the capital of the Inca, E. J. Brill, Leiden.

1977 "The Inca kinship system : a new theoretical view », in Ralph Bolton et Enrique Mayer, éds, Andean kinship and marriage, American Anthropological Association, Washington, DC, pp. 240-281.

1986 La civilisation inca au Cuzco, préface de Françoise Héritier, Presses universitaires de France, Paris.

1989 "What does the equation "mother's brother-wife's father" mean in Inca social organization? », in Henry J. M. Claessen, éd., Variant views : five lectures from the perspective of the "Leiden tradition " in cultural anthropology, Universiteit van Leiden, Faculteit der Sociale Wetenschappen, Vakgroep Culturele Anthropologie en Sociologie der Niet-Westerse Samenlevingen, Leiden, pp. 132-155, coll. " ICA Leiden » 84.

1997 «La política matrimonial incaica según Juan de Betanzos : un ejemplo implicando a los reyes Inca Roca y Yahuar Huacac », in Rafael Varón Gabai et Javier Flores Espinoza, éds, Arqueología, antropologia e historia en los Andes. Homenaje a María Rostworowski, Instituto de Estudios Peruanos, Banco Central de Reserva del Perú, Lima, pp. 289-300.

1998 «Espacio-tiempo y las panakas en la organización del Cuzco : hacia un modelo prehispánico », in Denise Y. Arnold, éd., Gente de carne y hueso. Las tramas de parentesco en los Andes. Parentesco y género en los Andes. II, CIASE/ILCA, La Paz, pp. 415-435.

2002 «La organización religiosa del sistema de panacas y memoria en el Cuzco Incaico ", in Jean-Jacques Decoster, éd., Incas e indios cristianos. Élites indigenas $e$ identidades cristianas en los Andes coloniales, CBC/IFEA/ Asociación Kuraka, Cuzco/Lima, pp. 19-38.

s. d. El calendario inca. Tiempo y espacio en la organización ritual del Cuzco, Fondo editorial del Congreso, Pontificia Universidad Católica del Peru, Universidad de San Marcos, Lima [sous presse]. 